\title{
A New Forecasting Method of Receiving Array Transducer State
}

\author{
Zhang Yang \\ Electronic Engineering College \\ Navy University of Engineering \\ Wuhan, China \\ E-mail: 18672325415@163.com
}

\author{
Wang Ping Bo \\ Electronic Engineering College \\ Navy University of Engineering \\ Wuhan, China \\ E-mail: 18672325415@163.com
}

\author{
Peng Cheng \\ Electronic Engineering College \\ Navy University of Engineering \\ Wuhan, China \\ E-mail: 992046452@qq.com \\ Jiang Li Zhi \\ Electronic Engineering College \\ Navy University of Engineering \\ Wuhan, China \\ E-mail: 18672325415@163.com
}

\begin{abstract}
This article is launched based on the target that to research the forecasting method of transducer state. Generally, the receive array of sonar is made up of several transducers according to one certain way, when the damaged quantity or degree of transducer reaches a specified level, there will have a great effect on the receive directivity of sonar, so that it needs to replace new transducer units. Up to now, there is no uniform forecasting method of transducer state. This article has implemented a new forecasting method of transducer state based on the performance estimate of the array: Firstly, the influence of insulation resistance is introduced into beamforming through ensuring the mathematic relationship between insulation resistance, working frequency and beamforming weighted coefficient of transducer; Secondly, after comparing sampling with standard of beam, technical factors is found to justice whether the beam directivity satisfy the requirement; Finally, to start transducer replacement, the status of it is estimated by investigating these index. The experiment result indicates that this method is effective.
\end{abstract}

Keywords- sonar; receiving array transducer; directivity; state forecasting; beamforming

\section{INTRODUCTION}

Generally, the receive array of sonar is made up of several transducers according to one certain way[1]. While the damage of one or a few units can't reduce the technical performance of sonar shapely, and it can work as normal. But when the damaged quantity or degree of transducer reaches a specified level, there will have a great effect on the receive directivity of sonar, so that it needs to replace new transducer units. Because of many human factors, there is no uniform forecasting method of transducer state.

The influence of insulation resistance is introduced into beamforming through ensuring the mathematic relationship between insulation resistance[2], working frequency and beamforming weighted coefficient[3] of transducer. After comparing sampling with standard of beam, technical factors is found to justice whether the beam directivity[4] satisfy the require. The damaged quantity and distribution of transducer in sonar main receive array, as well as the performance that transducer performance degradation effect on the sonar system, are studied in-depth analysis through computer simulation. By comparing the technical indicators of measuring beam and standard beam to determine directional meets the requirements to decide whether to replace the transducer, thus establishing the transducer state forecasting method[5].

\section{PROCESS OF FORECASTING METHOD}

In this article, state of the transducer array based on the received prediction main directivity, and the transducer is determined whether to replace by comparing the directional indicators. The process is shown in Fig .1. The situation is called standard situation when all the primitives are intact(when the weighting coefficients of beamforming are all 1), at this point the resulting beam is called the standard beam, and various directivity parameters called the standard parameters. In contrast, the situation is called measuring situation when there are primitive circumstances damaged(when the weighting coefficient of beamforming is between 0 and 1 ), at this point the resulting beam is called the measuring beam, and various directivity parameters called the measuring parameters.

Developing the main lobe deviation tolerance and side lobes height tolerance based on the requirement, and setting the test frequency and each under-measuring value of insulation resistance. The weighting coefficient of under-measuring resistance is acquired by the mathematic relationship between insulation resistance, working frequency and beamforming weighted coefficient of 
transducer. The weighting coefficient of standard beam is set as 1. Drawing measuring beam pattern and standard beam pattern, and comparing the main lobe width and the side lobe height[6] of the under-measuring beam with the main lobe width and the side lobe height of the standard beam, and to see whether the deviation is within the deviation tolerance. If there is one index that beyond the requirement, it is necessary to replace the transducer to ensure the receiving transducer of sonar to work properly.

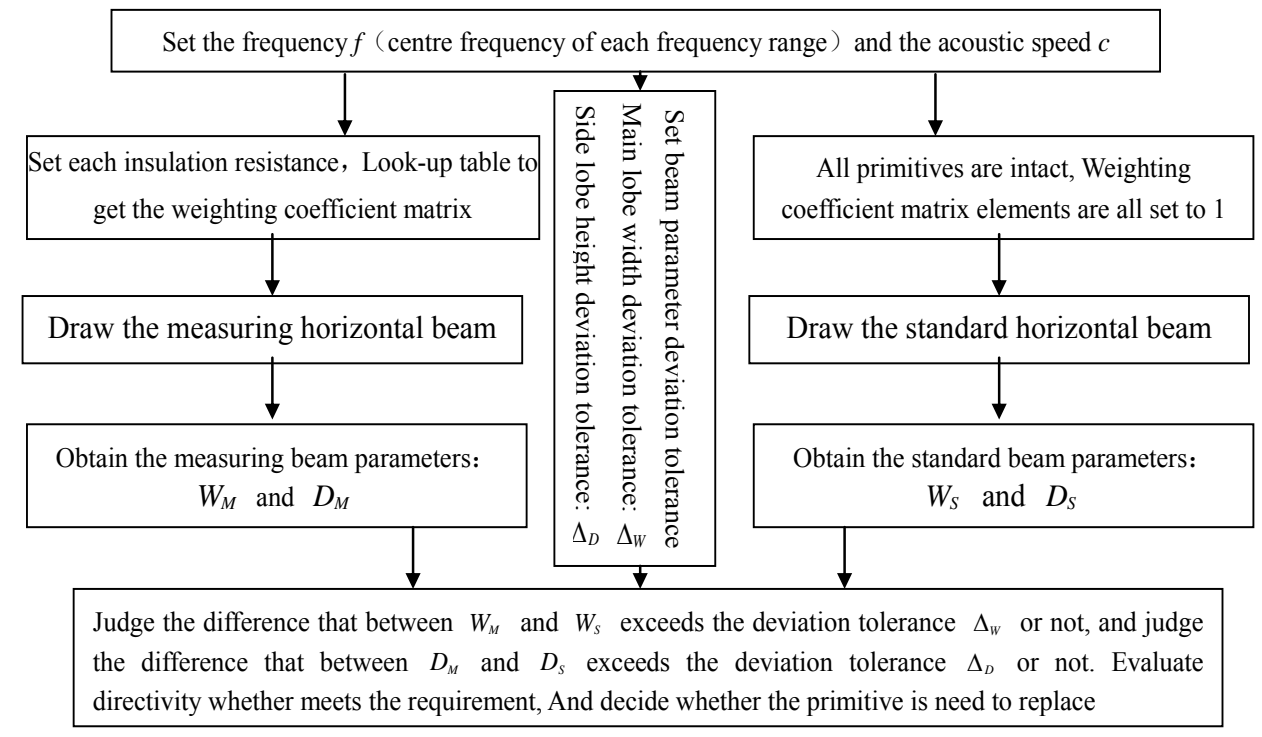

Figure 1. Process of forecasting method.

There are three key technologies that beamforming principle[6], the obtaining of weighting coefficient and the developing of key performance indicators which will be discussed as the following.

\section{KEY TECHNOLOGIES}

\section{A. Beamforming Algorithm Principle}

The $2 \mathrm{M}(\mathrm{M}=12)$ units uniform circular array sonar[7] is used as an example to illustrate the principle of horizontal beam forming algorithm[8]. The array model is shown in Fig .2

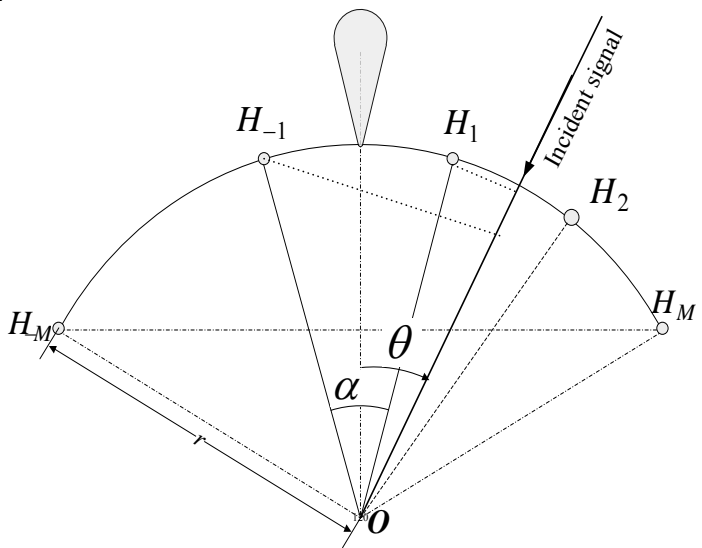

Figure 2. Beamforming of the $2 \mathrm{M}$ units uniform circular array.

$\theta$ represents the signal incident direction, $\theta_{0}$ indicates the direction of the beam control, $\alpha$ represents the angle between two adjacent primitives, the unit of all angles is rad, $r$ represents the radius of the arc. Setting the signal of point $O$ as: $s_{o}(t)=A \cos (2 \pi f \cdot t), f$ is the frequency, $t$ represents the time. The normalized directivity function is also called horizontal beam on the beam control $\theta_{0}$. It can be described as[4]:

$$
\begin{aligned}
B\left(\theta, \theta_{0}\right)= & \frac{1}{2 M}\left\{\left[\sum_{i=1}^{M}\left(w_{i} \cos \left(2 \pi f \Delta_{i}\right)+w_{-i} \cos \left(2 \pi f \Delta_{-i}\right)\right)\right]^{2}\right. \\
& \left.+\left[\sum_{i=1}^{M}\left(w_{i} \sin \left(2 \pi f \Delta_{i}\right)+w_{-i} \sin \left(2 \pi f \Delta_{-i}\right)\right)\right]^{2}\right\}^{1 / 2}
\end{aligned}
$$

Where, $\Delta_{i}=\tau_{i}(\theta)-\tau_{i}\left(\theta_{0}\right), \tau_{i}(\theta)$ is the delay that $H_{i}$ compares with point $O$. The expression of $\tau_{i}(\theta)$ follows as:

$$
\tau_{i}(\theta)=\frac{r \cos [\theta-(i-0.5) \alpha]}{c} \quad i=1,2, \mathrm{~L}, M
$$

Where, $c$ represents the speed of sound, $\Delta_{-i}=\tau_{-i}(\theta)-\tau_{-i}\left(\theta_{0}\right), \tau_{-i}(\theta)$ is the delay that $H_{-i}$ compares with point $O$. The expression of $\tau_{-i}(\theta)$ follows as:

$$
\tau_{-i}(\theta)=\frac{r \cos [\theta+(i-0.5) \alpha]}{c} \quad i=1,2, \mathrm{~L}, M
$$

$w_{i}$ and $w_{-i}$ represent the amplitude weighting coefficients of each array unit in the right and left sections.

\section{B. Obtaining the Weighting Coefficient}

The curve of receiving sensitivity and insulation resistance is fitted, by measuring the receiving sensitivity in different insulation resistance of transducers[2,4]. Calculating the mathematical model and the data table of 
receiving sensitivity according to insulation resistance in different frequency bands. The method of using transducer to measure the receiving sensitivity shows in Fig .3 .

The equivalent capacitance $\mathrm{c}$ of receiving transducer is $\mathrm{pF}$ degree. The equivalent resistance is so great that the resistances of different transducers can be equivalent by different external resistor[9]. As shown in formula (4):

$$
R=\left(R_{0}+\frac{1}{j 2 \pi f C}\right) / / R_{l}
$$

Where, $R_{0}$ represents the equivalent resistance of receiving transducer, $R_{l}$ represents external resistor, requirements of $R_{l} \gg R_{0}$. " //" represents parallel computing.

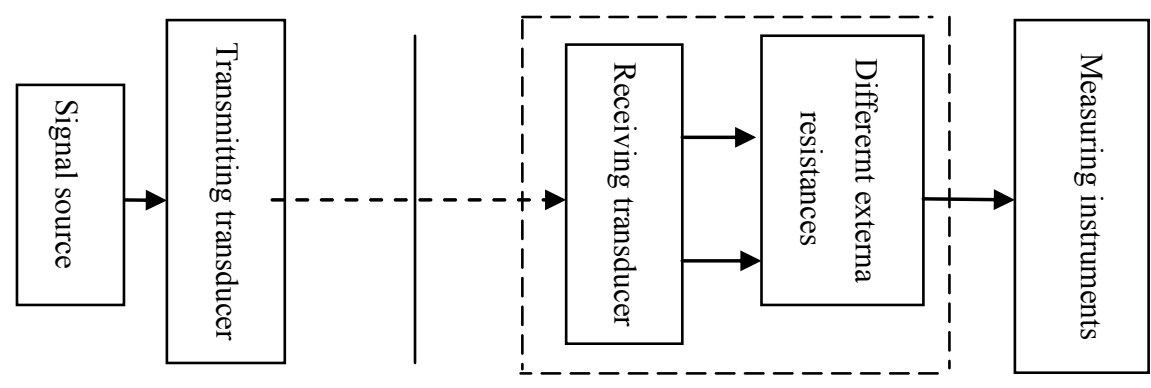

Figure 3. Process of forecasting method.

While the insulation resistance of transducer is large enough(standard resistance), it will normally play the role in beamforming. As well as the weighting factor is 1 . And taking it as the standard factor to compare the measuring value of each insulation resistance $\left(R_{m s r}\right)$ with the standard resistance value $\left(R_{s t d}\right)$ in a frequency $f$, and compare the receiving sensitivity $\beta_{m s r}$ with $\beta_{m s r}$ of the transducer(the unit is $\mathrm{dB})$. The weighting coefficient $w(f, R)$ which is corresponded to insulation resistance, can be calculation when the frequency has been determined, by the formula: $20 \lg w=\beta_{m s r}-\beta_{s t d}$. Such as, the frequency of a transducer is $4 \mathrm{~K} \Omega$, the value of insulation resistance is $1 \mathrm{KHz}$, the value of standard resistance is $100 \mathrm{~K} \Omega$, and the deviation of receiving sensitivity is $-2.2 \mathrm{~dB}$, and the weighting coefficient is $0.78[10]$. Using this method can get the table of frequency $\sim$ resistance value $\sim$ weighting coefficient.

In practical application, the weighting coefficient can be found in the table based on the insulation resistance $R$ and frequency $f$ of the current primitive. If there is no appropriate resistance can correspond to frequency, it can take the interpolation calculation method to acquire the weighting coefficient[11].

\section{Formulating the Key Index}

This article mainly evaluates that the deviation between the main lobe width $W_{M}$ and the standard value of horizontal beam is whether within the range of the deviation tolerance $\Delta_{W}$, and the deviation between the side lobe height $D_{s}$ and the standard value of horizontal beam is whether within the range of the deviation tolerance $\Delta_{D}$.

The main lobe width is the $-3 \mathrm{~dB}$ open-angle of the main lobe. It is positive number with the unit " $\circ$ ". Generally, the main lobe width should be as small as possible. The side lobe height is the deviation between the maximum of side lobe and the maximum of main lobe. It is negative number with the unit $\mathrm{dB}$. Generally, the side lobe height should be also as small as possible. And the deviation tolerance is the maximum permissible deviation between the measuring value of each index and the standard value.

The calculation method of the main lobe deviation is as follow: $E_{w}=W_{u}-W_{s}$. Generally, the damaged primitives can lead to larger main lobe width, and so $W_{s} \geq W_{s}, E_{w} \geq 0$. It can be able to determine whether the width of the main lobe meet the requirement by comparing $E_{W}$ and $\Delta_{W}$. It is considered not to meet the requirement, when $E_{W}>\Delta_{W}$, else it meet the requirement.

The calculation method of the side lobe height deviation $E_{D}$ can be defined as: $E_{D}=\left|\left(D_{M}-D_{s}\right) / D_{s}\right|$. Generally, the damaged primitives can lead to higher side lobe. It can be able to determine whether the height of the side lobe meet the requirement by comparing $E_{D}$ and $\Delta_{D}$. It is considered not to meet the requirement, when $E_{D}>\Delta_{D}$, else it meet the requirement.

\section{THE SIMULATION}

This article adopts the uniform circular array sonar with 24 primitives. When it works on the working frequency[10], the default parameter index is as follows: the standard value of main lobe width is 19 , the deviation tolerance is \pm 4 ; and the standard value of side lobe is -8.1 , the deviation tolerance is $15 \%$.

Supposing that there is a primitive damaged in the uniform circular array sonar, the fitting horizontal beam shows in Fig .4 The horizontal axis is horizontal, and the unit of it is "० ". The ordinate is the logarithm amplitude of the beam, and the unit of it is $\mathrm{dB}$. The solid line represents the measuring beam, and the dotted line represents the standard beam. (This description is also applicable to the following diagram). 
In Fig .4, the measuring value of the main lobe width is 19.5 , the main lobe deviation: $E_{W}=0.5<4$; the measuring value of the side lobe height is -7.9 , the side lobe deviation : $E_{D}=2.5 \%<15 \%$, all the index meets the requirements, so the directivity also meets the requirement, and the sonar can work normally, there is no transducer needs to be replaced.

When there are three continuous damaged primitives in the sonar, the fitting horizontal beam shows in Fig .5. In this case, the measuring value of the main lobe width is 17 , the main lobe deviation: $E_{w}=2<4$, it doesn't meet the requirement; the measuring value of the side lobe height is -5.9, the side lobe deviation : $E_{D}=27 \%>15 \%$, it doesn't meet the requirement. At this time, the performance of directivity declines so seriously that the transducers need to be replaced.

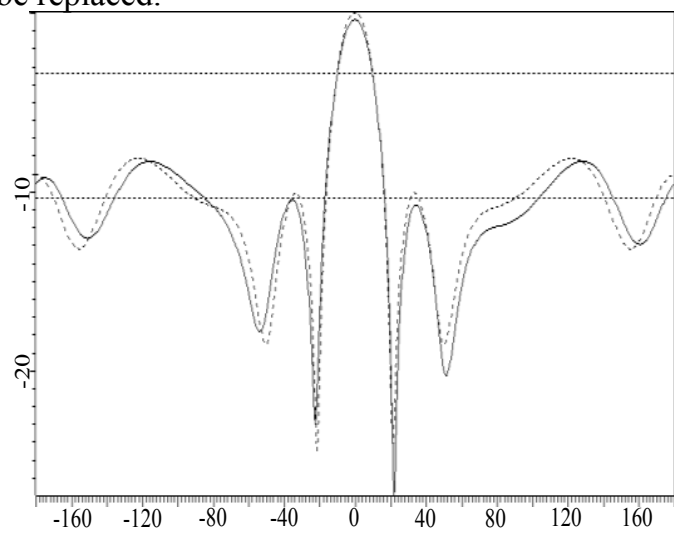

Figure 4. Performance analysis test 1.

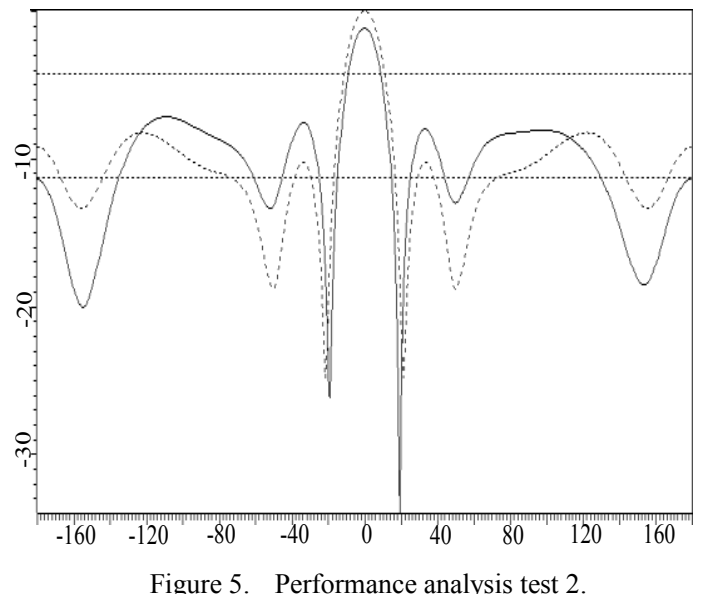

The above discussion is a certain (some) primitive completely damaged situation (the insulation resistance is 0 , and the weighting coefficient is also 0 ). In the situation that primitive is not completely damaged, the weighting coefficient is not 0 but less than 1. The corresponding forecast results should be a certain extent between the results in the above situations.

\section{CONCLUSION}

In this article, a uniform circular array sonar with 24primitives is taken as an example. The damaged quantity and distribution of transducer in sonar main receive array, as well as the performance that transducer performance degradation effect on the sonar system, are studied indepth analysis. Ensuring the mathematic relationship between insulation resistance, working frequency and beamforming weighted coefficient of transducer. Founding the technical factors to justice whether the beam directivity satisfy the requirement. And the forecasting method of transducer state is proposed. This method has a strong practical significance in forecasting the replacement of sonar transducer.

\section{ACKNOWLEDGMENT}

This research is partially supported by the project of National Natural Science Foundation of China with grant No.51109218

\section{REFERENCES}

[1] Luan Gui Dong, Zhang Jin Zhe, Wang Ren Qian, Piezoelectric Transducer And Transducer Array, 1st ed, Bei Jing: Peking University Press, 2005, pp 326-350.

[2] Lin Feng, "Study on the Intelligent detection of Multiple sonar transducer insulation resistance and equivalent capacitance," China Ship Repair, vol. 22, Dec. 2009, pp. 33-35

[3] Liu Cong Feng, Research on Robust Adaptive Beamforming and Space-Time Adaptive Processing Algorithms. Xi An: Xi An Electronic and Science University, 2008.

[4] Lan Jun, "A General Method of Directivity Synthesis," Acta Acustica, vol. 8, Jul. 1983, pp. 210-219.

[5] Liu Lei, Liu Wang Suo, Wei Yu Hua, "New Prediction Method of Performance State for Receiving Transducer Array," Ship Electronic Enineering, vol. 5, Mar. 2008, pp. 170-173.

[6] Zhang Cheng, Chen Ke An, Yang Zhi Xing, "Performance Analysis of Beamforming for Circular Array with Rigid Cylindrical Baffle," Acta Acustica, vol. 35, Jan. 2010, pp. 68-75.

[7] Qiao Wen Xiao, Che Xiao Hua, Ju Xiao Dong, Chen Xue Lian, "Acoustic Logging Phased are Array and Its Radiation Directivity," Chinese Journal of Geophysics, vol. 51, May. 2008, pp. 939-946.

[8] Li Qi Hu, Digital Sonar Design Principles, 1st ed, He Fei: Anhui Education Press, 2003, pp 413-415.

[9] Teng Duo, Study on The Technology of Sonaer Transducer and Transucer Array. Xi An: Northwestern Polytech University, 2004.

[10] Liu Wang Suo, The Performance Evaluation System Technical Report of A Certain Type Integrated Sonar Main Receiving Transducer. Wu Han: Navy University of Engineering, 2006.

[11] Hu Guang Shu, Digital Signal Proessing, 2st ed, Bei Jing:Peking University Press, 2003, pp 413-415. 